\title{
Performance Issues Associated With WDM Optical Systems Using Self-Seeded Gain Switched Pulse Sources Due to Mode Partition Noise Effects
}

\author{
P. Anandarajah, Student Member, IEEE, L. P. Barry, Member, IEEE, and A. Kaszubowska, Student Member, IEEE
}

\begin{abstract}
Bit-error-rate measurements have been carried out on a four-channel wavelength division multiplexed setup using tunable self-seeded gain-switched pulse sources. These measurements demonstrate the degradation in overall system performance, due to mode partition noise, as the sidemode suppression ratio of the self-seeded gain-switched optical pulse sources is reduced. The results also show that the constraints on the minimum sidemode suppression ratio required increase with the number of channels in the system.
\end{abstract}

Index Terms-Optical fiber communications, optical pulse generation, mode partition noise, semiconductor laser, wavelength-division multiplexing.

\section{INTRODUCTION}

A S OPTICAL communication systems move to line rates of $40 \mathrm{~Gb} / \mathrm{s}$ and beyond, it becomes more likely that return-to-zero (RZ) coding will be used for data transmission, as it is easier to compensate for dispersion and nonlinear effects in the fiber by employing soliton-like propagation [1]. In addition to this development, the use of wavelength tunability in optical networks is being explored as a way to provide dynamic provisioning in the next generation of photonic systems [2]. Taking into account these moves toward tunable optical systems employing RZ coding, it is obvious that the development of a wavelength tunable source of short optical pulses will be of paramount importance for future WDM, optical time division multiplexed (OTDM), and hybrid WDM/OTDM optical communication systems [3].

Self-seeding of a gain-switched Fabry-Pérot (FP) laser is one of the most reliable techniques available to generate wavelength tunable optical pulses [4], [5]. An important characteristic of these self-seeded gain-switched (SSGS) sources is the variation in the sidemode-suppression-ratio (SMSR) as the wavelength is tuned [4], [5], as this may ultimately affect their usefulness in optical communication systems. Recent work has demonstrated how this SMSR variation greatly affects the noise induced on a single pulse source as the pulses propagate through optical fiber and an optical filter [6]. In addition, by using two SSGS pulse sources at different wavelengths, we have examined the noise induced on one of the pulse sources due to a variation in SMSR of the other SSGS source [7].

\footnotetext{
Manuscript received February 12, 2002; revised April 11, 2002.

The authors are with the School of Electronic Engineering, Dublin City University, Dublin 9, Ireland (e-mail: anandara@eeng.dcu.ie).

Publisher Item Identifier S 1041-1135(02)06086-X
}

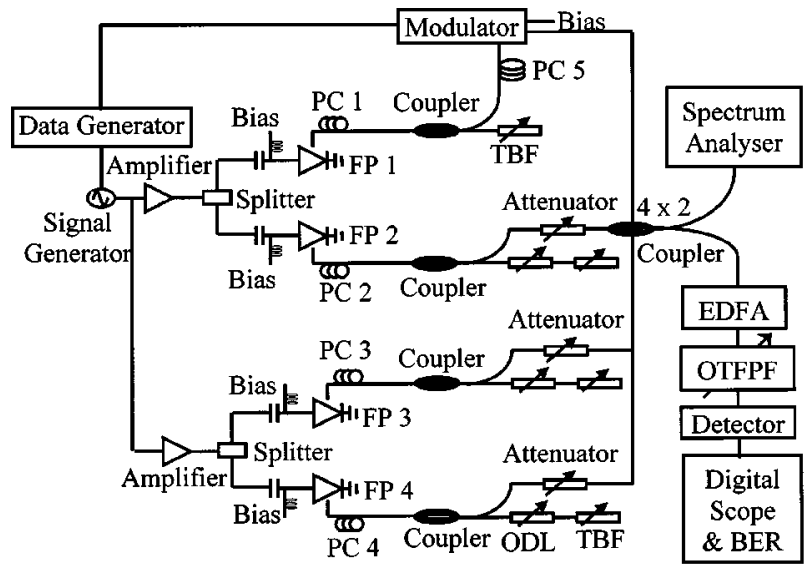

Fig. 1. Experimental setup for examining the effects of SMSR variation in a WDM system using self-seeded gain-switched pulse sources.

In this paper we experimentally investigate the system performance, by using bit-error-rate (BER) measurements, of a four-channel WDM system employing SSGS pulse sources, as the SMSR of the sources is varied. The cross channel interference due to mode-partition-noise results in significant power penalties in the system as the SMSR of the pulse sources are degraded. Our results also demonstrate that as the number of channels in a WDM system using SSGS pulse sources increases, the specifications on the required SMSR become more stringent.

\section{EXPERIMENTAL SETUP}

Fig. 1 shows our experimental setup. The FP lasers used were commercial 1.55- $\mu \mathrm{m}$ InGaAsP devices, with threshold currents around $20 \mathrm{~mA}$, and longitudinal mode spacings of $1.1 \mathrm{~nm}$. The four lasers were gain-switched by applying dc bias currents of around $25 \mathrm{~mA}$ in conjunction with $2.5-\mathrm{GHz}$ electrical sinusoidal signals (with powers of $24 \mathrm{dBm}$ ), to each diode. Self-seeding of the diode FP1 was achieved by using an external cavity containing a polarization controller (PC), a 3-dB coupler, and a tunable Bragg grating with a bandwidth of $0.4 \mathrm{~nm}$. The external cavities for self-seeding FP2, FP3 and FP4 contained additional tunable optical delay lines (ODL) [7].

To achieve optimum SSGS pulse generation from FP1, the grating was tuned to reflect one of the laser modes (at $1552.6 \mathrm{~nm})$, and the frequency of the sinusoidal modulation was then varied $(\sim 2.4836 \mathrm{GHz})$ to ensure that the signal re-injected into the laser arrives at the correct time. For SSGS operation of all the other FPs 2-4, each of the Bragg gratings 

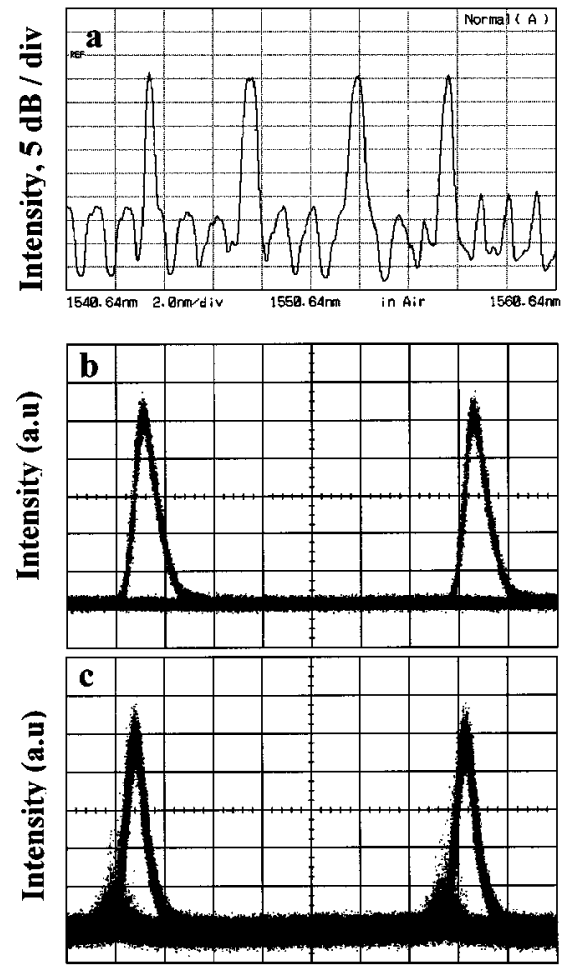

Time, $60 \mathrm{ps} / \mathrm{div}$

Fig. 2. (a) Optical spectrum of the composite wavelength signal after fiber coupler. (b) Back-to-back eye diagram for $1552.6 \mathrm{~nm}$ data channel. (c) Received eye diagram of 1552.6-nm data channel with SMSR of other pulse sources in the WDM signal set to $20 \mathrm{~dB}$.

were tuned to reflect laser modes at 1544.1, 1548.1, $1556.3 \mathrm{~nm}$, respectively. The ODL was varied to ensure that the signals fed back from the gratings arrive at the correct time. In addition to tuning the grating, the feedback can be adjusted, (and thus, the SMSR on the output pulses varied), by using the polarization controllers (PC) at the laser output.

A $2^{11}-1$ pseudorandom data signal from a pattern generator, at a bit rate of $2.5 \mathrm{~Gb} / \mathrm{s}$, was then used to modulate the 1552.6-nm pulse train. The resulting $2.5-\mathrm{Gb} / \mathrm{s}$ RZ data signal from the modulator was then coupled together with the other three pulse trains with the aid of a $4 \times 2$ fiber coupler. The other three pulse train signals were attenuated before the coupler to ensure that the power level in each wavelength signal was the same after the coupler. The composite signal was then amplified before the 1552.6-nm data signal was filtered out using a tunable filter with a bandwidth of $0.7 \mathrm{~nm}$. The received data signal was then detected using a 50-GHz p-i-n photodiode, before a $50-\mathrm{GHz}$ oscilloscope was used to examine the received eye diagrams, and an error analyzer was used for BER measurements.

\section{RESULTS}

Fig. 2(a) displays the optical spectrum of the composite signal after being combined together using the fiber coupler, (with the feedback from the gratings optimized using the polarization controllers). The $3-\mathrm{dB}$ bandwidth of the $2.5-\mathrm{GHz}$ pulse sources varied from 0.2 to $0.3 \mathrm{~nm}$, and the pulse width varied from about 18 to 26 ps (measured using oscilloscope and deconvolution with response time of measurement setup). The optimized SMSR of each source was about $30 \mathrm{~dB}$.

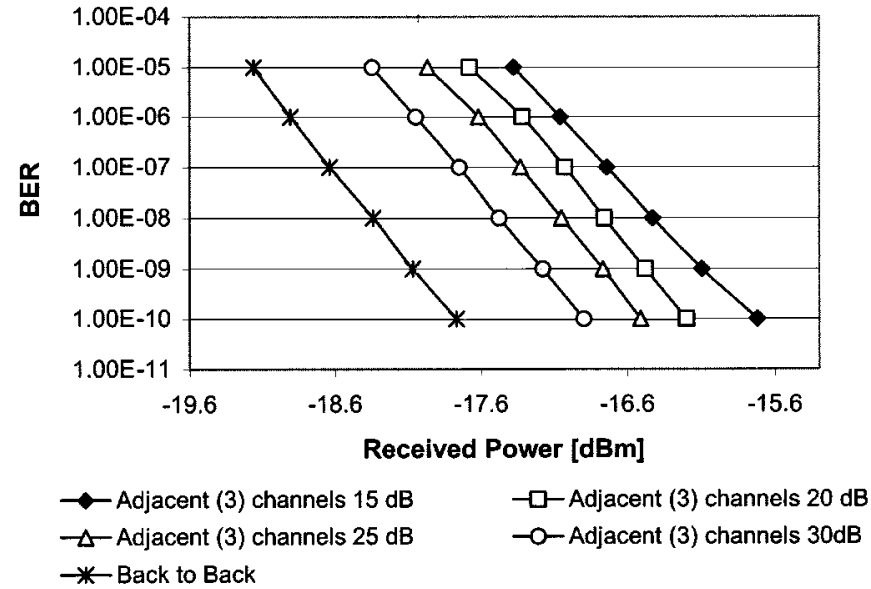

Fig. 3. BER versus received power for back-to-back case, and when the SMSR of adjacent pulse sources were set to $30,25,20$, and $15 \mathrm{~dB}$.

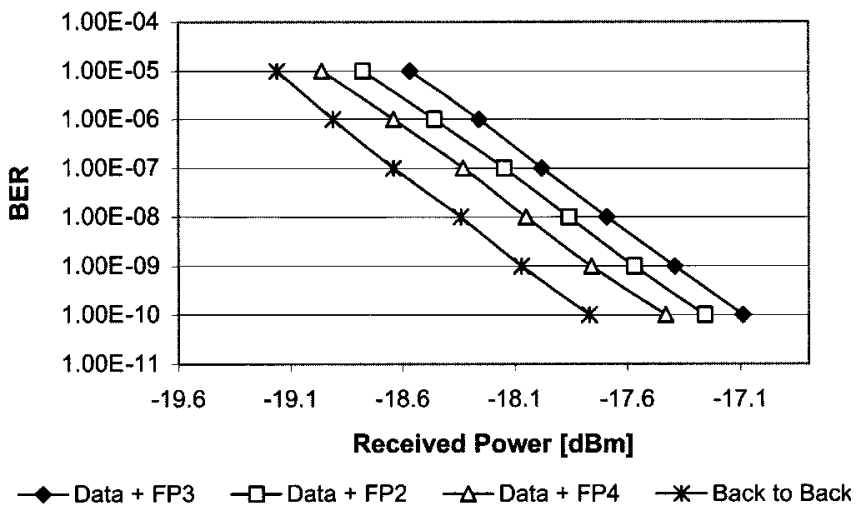

Fig. 4. BER versus received power for back-to-back case, and when data signal was multiplexed individually with each pulse source (SMSR maintained at $30 \mathrm{~dB})$.

Fig. 2(b) displays back-to-back eye diagram of the 1552.6-nm data signal (when the three adjacent pulse sources were momentarily turned off). To determine the effect of SMSR variations on the four-channel WDM system, we proceeded to vary the SMSR of the adjacent pulse sources using the PCs, while the optical filter was tuned to select out the 1552.6-nm data channel (which had maximum SMSR $\sim 30 \mathrm{~dB}$ maintained throughout). Fig. 2(c) displays one of the results, and is the received eye diagram (of 1552.6-nm data channel) when the SMSR of the three pulse sources were set to $20 \mathrm{~dB}$. The increased noise of the eye diagram in comparison with Fig. 2(b) can be clearly seen.

As the SMSR of the three pulse sources were varied in the experimental arrangement, measurements of the BER versus received optical power, for the 1552.6-nm data channel were recorded. Fig. 3 displays BER vs. received power curves for the back-to-back case, and when the SMSR of the three pulse sources in the WDM signal, were set to $30,25,20$, and $15 \mathrm{~dB}$. The power penalty introduced by each of these settings was 0.9 , 1.3, 1.6, and $2 \mathrm{~dB}$, respectively. We then examined the effect of multiplexing the 1552.6-nm data channel with each one of the pulse sources individually, with the SMSR of the pulse source maintained at $30 \mathrm{~dB}$. As we can see from Fig. 4, the power penalty presented due to the introduction of one source, with a SMSR of $30 \mathrm{~dB}$, can vary from 0.3 to $0.7 \mathrm{~dB}$, for a BER of $10^{-9}$. 


\section{DisCUSSIONS AND CONCLUSION}

The degradation in BER of the 1552.6-nm data signal as we introduce additional wavelength channels, and reduce the SMSR of these pulse sources, is due to the mode partition effect [8], [9]. This effect is basically a fluctuation of the energy in each laser mode with time, due to a constant transfer of energy between the modes. For a single-mode laser with a large SMSR, the power in the sidemodes is negligible, thus the power fluctuation of the main mode is negligible. However, as the SMSR decreases the power fluctuation of the main mode, and the sidemodes, may become nonnegligible.

With the optical filter tuned to select out the 1552.6-nm data signal, if the SMSR of the adjacent pulse soures is so large that the fluctuation in power of their sidemodes (around $1552.6 \mathrm{~nm}$ ) is negligible, then there will be no power penalty for the received data signal. However, as the SMSR of the adjacent channels is degraded, the system performance decreases, due to the increased fluctuation in power of the sidemodes (as presented in Fig. 3). The overall power penalty experienced for the WDM system is due to the cumulative effect of the power fluctuation in the sidemodes, which are at the same wavelength as the filtered data signal. Table I displays the power penalties introduced (relative to back-to-back measurement) as the 1552.6-nm data channel is multiplexed with the various combinations of the three pulse sources (which have their SMSR maintained at $30 \mathrm{~dB}$ ). Clearly as the number of channels increases, so does the power penalty, however the increase in power penalty is determined by which of the sources are multiplexed with the data channel. This is because the FP lasers used to generate the pulses have different gain curves. The result of this is that even though the SMSR of all the sources is maintained at $30 \mathrm{~dB}$, the powers in the sidemode, which lies at the same wavelength as the data signal (and cause the power penalty), are different for each pulse source. This effect is clearly seen by examining the system performance introduced when the data signal is multiplexed with just one source (Fig. 4). The degradation in system perfomance in this case is dependent on which pulse source is multiplexed with the data (due to the different gain curves of the lasers). By examining the spectra from the three different pulse sources, we can determine the difference in power levels between the sidemode of each source at the wavelength of the data signal $(1552.6 \mathrm{~nm})$, and the power level in the data signal. The relative differences are $31.9 \mathrm{~dB}, 33.6 \mathrm{~dB}$, and $35.3 \mathrm{~dB}$ for sources FP3, FP2, and FP4 respectively. We can thus see from these values, and from Fig. 4, that as the difference in power level between the data signal and the sidemode of the multiplexed source at the data signal wavelength decreases, the power penalty in the system increases, as expected.

In conclusion, we have shown how the SMSR of wavelength tunable SSGS pulse sources affects the performance of WDM communication systems which employ such sources. As the SMSR of one or more sources in a WDM system becomes de-
TABLE I

Power Penalties Relative to Back-to-Back Measurement,

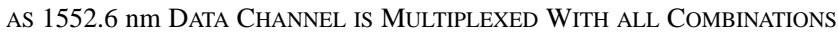
of THE THReE PUlse Sources (SMSR MaINTAINEd AT $30 \mathrm{~dB}$ ).

\begin{tabular}{c|c}
\hline $\begin{array}{c}\text { Data Signal Multiplexed } \\
\text { with pulse source : }\end{array}$ & $\begin{array}{c}\text { Power Penalty } \\
\text { Introduced (dB) }\end{array}$ \\
\hline FP2 & 0.5 \\
\hline FP3 & 0.7 \\
\hline FP4 & 0.3 \\
\hline FP2 + FP3 & 0.8 \\
\hline FP3 + FP4 & 0.7 \\
\hline FP2 + FP4 & 0.6 \\
\hline FP2 + FP3 + FP4 & 1.0 \\
\hline
\end{tabular}

graded, then the interaction of the mode partition effect with spectral filtering will result in increased noise on all the received wavelength channels in the system. This additional noise introduces a power penalty into the overall system performance. In addition, as the number of channels in a WDM system using SSGS sources increases, the minimum required SMSR of each source, such that it does not affect system performance, will increase.

\section{REFERENCES}

[1] R. Ludwig, U. Feiste, E. Dietrich, H. G. Weber, D. Breuer, M. Martin, and F. Küppers, "Experimental comparison of $40 \mathrm{Gbit} / \mathrm{s} \mathrm{RZ}$ and NRZ transmission over standard single mode fiber," Electron. Lett., vol. 35, pp. 2216-2218, 1999.

[2] C.-K. Chan, K. L. Sherman, and M. Zirngibl, "A fast 100-channel wavelength-tunable transmitter for optical packet switching," IEEE Photon. Technol. Lett., vol. 13, pp. 729-731, July 2001.

[3] T. Morioka, H. Takara, S. Kawanishi, O. Kamatani, K. Takiguchi, K. Uchiyama, M. Saruwatari, H. Takahashi, M. Yamada, T. Kanamori, and H. Ono, "1 Tbit/s (100 Gbit/s times 10 channel) OTDM/WDM transmission using a single supercontinuum WDM source," Electron. Lett., vol. 32, pp. 906-907, 1996.

[4] L. P. Barry, R. F. O' Dowd, J. Debeau, and R. Boittin, "Tunable transform limited pulse generation using self-injection locking of a FP laser," IEEE Photon. Technol. Lett., vol. 5, pp. 1132-1134, Oct. 1993.

[5] C. Shu and S. P. Yam, "Effective generation of tunable single- and multiwavelength optical pulses from a Fabry-Pérot laser diode," IEEE Photon Technol. Lett., vol. 9, pp. 1214-1216, Sept. 1997.

[6] L. P. Barry and P. Anandarajah, "Effect of side mode suppression ratio on the performance of self-seeded, gain-switched optical pulses in lightwave communications systems," IEEE Photon Technol. Lett., vol. 11, pp. 1360-1363, Nov. 1999.

[7] _ "Cross-channel interference due to mode partition noise in WDM optical systems using self-seeded gain-switched pulse sources," IEEE Photon Technol. Lett., vol. 13, pp. 242-244, Mar. 2001.

[8] N. H. Jensen, H. Olesen, and K. E. Stubkjaer, "Partition noise in semiconductor lasers under CW and pulsed operation," IEEE J. Quantum Electron., vol. 23, pp. 71-79, Jan. 1987.

[9] D. Curter, P. Pepeljugoski, and K. Y. Lau, "Noise properties of electrically gain-switched $1.5 \mu \mathrm{m}$ DFB lasers after spectral filtering," Electron. Lett., vol. 30, pp. 1418-1419, 1994. 